\section{MS21-P4 On the stacking disorder of an organic acid}

Christian Czech ${ }^{1}$, Edith Alig ${ }^{1}$, Kristoffer E. Johansson ${ }^{2}$, Jan Bats ${ }^{1}$, Martin U. Schmidt ${ }^{1}$

1. Goethe-University, Institute of Inorganic and Analytical Chemistry, Max-von-Laue-Straße 7, 60438 Frankfurt am Main, Germany

2. University of Copenhagen, Institute of Pharmacy, Universitetsparken 2, DK-2100 Copenhagen, Denmark

email: cczech@chemie.uni-frankfurt.de

Crystals of the $\alpha$ - and $\beta$-phase of 2-aminohexanoic acid $\left(\mathrm{C}_{6} \mathrm{H}_{13} \mathrm{NO}_{2}\right)$ frequently show diffuse streaks parallel to $c^{*}$, which indicates a stacking disorder in the layer structure. Order-disorder (OD) theory is used to derive possible stacking sequences. Lattice-energy minimisations by force fields and dispersion-corrected density functional theory (DFT-D) were performed on a set of ordered model structures. The calculated energies depend not only on the arrangement of neighbouring layers, but also of next-neighbouring layers. From the DFT-D energies stacking probabilities were calculated. According to the calculated stacking probabilities large models containing 100 double-layers were constructed. Their simulated diffraction patterns show sharp reflections for $\mathrm{h}+\mathrm{k}=2 \mathrm{n}$ and strong diffuse streaks parallel to $c^{*}$ through all reflections with $\mathrm{h}+\mathrm{k}=2 \mathrm{n}+1$, which is in good agreement to experimental investigations.

Keywords: stacking disorder, lattice energy minimisations, force fields, DFT-D,

\section{MS21-P5 Neutron diffraction study of the deformation behavior of Mg-alloy-based composites}

Gergely Farkas ${ }^{1}$, Kristián Máthis ${ }^{2}$, Ján Pilch ${ }^{1}$, Peter Minárik ${ }^{2}$, Petr Lukášl $^{1}$

1. Nuclear Physics Institute, ASCR, v. v. i.

2. Charles University in Prague, Department of Physics of Material, Czech Republic

email: farkasgr@gmail.com

Deformation mechanisms in $\mathrm{Mg}-\mathrm{Al}-\mathrm{Ca}$ alloy reinforced with short alumina fibres (30 wt. \%) were studied in-situ using neutron diffraction method. Ceramic fibres were homogeneously distributed in the matrix and are arranged to parallel planes, inside which they have random orientations. Two type of sample were investigated: samples with parallel fibre plane orientation and perpendicular fibre plane orientation with respect to the loading axis. The fibres plane orientation was found to be a key parameter, which influences the acting deformation processes, such as twinning or dislocation slip.

Deformation curves shows that parallel fibre plane orientation has stronger influence on the increment of yield stress and tensile strength $(600 \mathrm{MPa})$, that the perpendicular fibre orientation. Concurrently, the elongation is smaller. Mechanical properties of composite shows strong degradation at $200{ }^{\circ} \mathrm{C}$ and enhanced ductility up to $15 \%$ strain.

Results from in-situ neutron diffraction shows the lattice strain changes in the matrix and also in the reinforcement phase depending on macroscopic compressive deformation and stress. In case of parallel fibre plane orientation the increment of compressive lattice strain is lower in the matrix and higher in the fibres in comparison to perpendicular fibre orientation. This is a direct proof of Shear-lag model according to which the load transfer is more effective, when fibres close a small angle with the operating load.

At elevated temperature regardless of the fibre plane orientation, above $1 \%$ of deformation matrix`s lattice strain start to show inverse behaviour. Compressive strain in the matrix rapidly decreases with compressive deformation, without a significant loss of macroscopic stress.

Keywords: metal matrix composite, internal lattice strain, neutron diffraction 